Meta

Journal des traducteurs

Translators' Journal

\title{
La terminologie, pour quoi faire?
}

\section{Amal Jammal}

Volume 25, numéro 4, décembre 1980

URI : https://id.erudit.org/iderudit/003240ar

DOI : https://doi.org/10.7202/003240ar

Aller au sommaire du numéro

Éditeur(s)

Les Presses de l'Université de Montréal

ISSN

0026-0452 (imprimé)

1492-1421 (numérique)

Découvrir la revue

Citer cet article

Jammal, A. (1980). La terminologie, pour quoi faire? Meta, 25(4), 467-469.

https://doi.org/10.7202/003240ar

Ce document est protégé par la loi sur le droit d'auteur. L'utilisation des services d'Érudit (y compris la reproduction) est assujettie à sa politique d'utilisation que vous pouvez consulter en ligne.

https://apropos.erudit.org/fr/usagers/politique-dutilisation/
Cet article est diffusé et préservé par Érudit.

Érudit est un consortium interuniversitaire sans but lucratif composé de l'Université de Montréal, l'Université Laval et l'Université du Québec à Montréal. Il a pour mission la promotion et la valorisation de la recherche. https://www.erudit.org/fr/ 


\section{PROBLÈMES ET SOLUTIONS}

\section{LA TERMINOLOGIE, POUR QUOI FAIRE?}

A l'instar de M. Jourdain qui faisait de la prose sans le savoir, le traducteur ne fait-il pas, à un moment donné de l'acte traductionnel ( $\mathrm{sic}$ ), de la terminologie sans le savoir?

$\mathrm{Au} 23^{\mathrm{e}}$ congrès de l'Association des professeurs de français des universités canadiennes, qui s'est tenu à l'Université du Québec à Montréal du 28 au 31 mai 1980, plus précisément au cours de l'atelier sur la pédagogie de la traduction, M. Darbelnet s'est étonné que l'on fasse de la terminologie une discipline distincte de la traduction, alors que de toute évidence elle en est une partie intégrante. Selon M. Darbelnet, si l'on ampute ainsi le processus de la traduction de sa composante "terminologie» et si, comme cela semble se dessiner, on fait de même de sa composante «rédaction», que restera-t-il de la traduction?

Nous tenterons de répondre à cela en faisant d'abord une distinction qui nous semble essentielle. On ne peut placer sur le même pied rédaction et terminologie. En effet, si à la rigueur le traducteur peut se permettre de n'être qu'un peu terminologue, en revanche, il ne peut être un peu rédacteur; il sera bon rédacteur ou ne sera pas traducteur. Voilà toute la différence.

Un programme de traduction qui ne ferait qu'une place relativement restreinte à la rédaction risquerait fort de manquer son but, soit la formation de bons traducteurs. Par contre, on peut concevoir un programme qui ne donne à l'apprenti traducteur que des éléments de terminologie suffisants pour lui permettre de se sortir d'une impasse, le cas échéant. Cependant, un approfondissement des connaissances dans le domaine terminologique, qui nécessite une démarche pédagogique différente de celle de la traduction et l'acquisition de méthodes de travail particulières, demanderait plus de temps qui n'en peut raisonnablement offrir un programme de traduction de trois ans. Il est donc probablement plus logique, à cause de la divergence des moyens, des objectifs et même des aptitudes requises, de faire de ces deux domaines des disciplines distinctes.

La réflexion de $M$. Darbelnet se justifie à la rigueur si l'on pense à la traduction générale. Il en va tout autrement quand on aborde la traduction 
spécialisée. Nous prendrons pour exemple la traduction scientifique où l'on peut, pour paraphraser Julien Green, dire que la science vole et les mots vont à pied. En effet, dans le domaine scientifique, l'évolution de certaines disciplines est si rapide et les découvertes de techniques nouvelles si nombreuses que les néologismes se créent à une allure que les dictionnaires sont incapables de suivre. Nombreux sont les chercheurs anglophones qui, placés devant des réalités nouvelles, fabriquent des mots sans souci des balises lexicographiques, grammaticales ou autres, s'autorisant pour ce faire du prétendu laxisme de la langue anglaise. Que fera alors le traducteur? S'il est honnête, il oubliera que time is money et s'appliquera à dépouiller les dernières revues scientifiques pour découvrir qu'en réalité sont nés non pas un mais deux ou peut-être trois équivalents français du néologisme anglais. Qui lui dira lequel est le plus approprié? Nous soumettrons ici deux cas concrets. On a vu depuis quelque temps apparaitre deux expressions anglaises nouvelles: invasive - et non invasive - procedure. Invasive existe dans un dictionnaire médical unilingue anglais qui donne :

1. Pertaining to invasion. 2. Tending to invade healthy cells or tissues; said of microorganisms or tumors ${ }^{1}$.

Dans un autre dictionnaire médical anglais, seule la forme nominale de invasive, soit invasion, figure avec la définition suivante:

1. The attack or onset of a disease. 2. The simple harmless entrance of bacteria into the body or their deposition in the tissues as distinguished from infection. 3. The infiltration and active destruction of surrounding tissue, a characteristic of malignant tumors ${ }^{2}$.

Le dictionnaire unilingue français, pour sa part, est plus laconique :

Invasion : période de début d'une maladie, depuis l'installation des symptômes jusqu'à la période d'état ${ }^{3}$.

Enfin, le dictionnaire d'équivalences propose :

Invasive; envahissant ${ }^{4}$.

Point n'est besoin d'être traducteur ou terminologue pour se rendre comte qu'on ne peut rendre invasive procedure par technique envahissante. En fait, on finit par comprendre, d'après les textes où ces termes apparaissent, qu'il s'agit de techniques d'exploration diagnostique qui exigent l'introduction d'un instrument à l'intérieur de l'organisme par des moyens chirurgicaux. Pour décrire ces techniques diagnostiques, certains auteurs francophones parlent de techniques sanglantes ou non sanglantes ${ }^{5}$. D'autres, auxquels peut-être répugnent ces termes à forts relents de boucherie, préfèrent parler de techniques

1. Blakinston's Gould Medical Dictionary, $3^{\mathrm{e}}$ edition, New York, McGraw Hill, 1972.

2. Dorland's Illustrated Medical Dictionary, $25^{\mathrm{e}}$ edition, London, Saunders, 1974. 3. A. Manuila, L. Manuila et coll., Dictionnaire français de médecine et de biologie, Paris,

4. W. J. Gladstone, Dictionnaire anglais-français des sciences médicales et paramédicales, SaintHyacinthe, Edisem, 1978.

5. J. Pernod, «L'écographie par ultrasons en cardiologie", L'encyclopédie médico-chirurgicale, Paris, Édition technique, p. $1-32006$ à 10 . 
traumatiques ou atraumatiques ${ }^{6}$; peut-être aussi, en ce faisant, pensent-ils à certains cathétérismes qui, tout en constituant une «invasion» en quelque sorte de l'organisme, ne sont pas nécessairement sanglants mais sont néanmoins traumatiques. Un problème analogue se pose pour open surgical procedure où l'on a, ici encore, comme équivalent, le choix entre l'intervention sanglante et l'inconcevable intervention à ciel ouvert aux fausses allures de piquenique. Quoi choisir et sur quelles bases? N'est-ce pas au terminologue, qui dispose du temps, des moyens et de la compétence nécessaires, que revient la tâche d'étudier la fréquence d'apparition des termes, d'analyser leur contenu notionnel et de décider de leurs valeurs respectives; en somme, d'indiquer ce qui doit être retenu et pourquoi?

Laisser au traducteur pressé (que l'on nous pardonne ce pléonasme) le soin de faire le choix, c'est exposer la langue de spécialité à une véritable gabegie synonymique dont nous avons déjà un exemple dans la langue médicale.

Est-ce à dire qu'il faut évacuer totalement la pédagogie de la traduction de son élément terminologie, sous prétexte que l'on aura promu cette dernière au rang de discipline distincte? Non, car il est indéniable que le traducteur doit être aussi un peu terminologue. Néanmoins, la terminologie, discipline autonome, suppose une démarche d'apprentissage particulière, l'acquisition d'une méthode spécifique de recherche, une bonne connaissance des sources et des moyens de documentation, une étude systématique des termes, le tout nécessitant une période de formation appropriée. Le terminologue ainsi formé pourra répondre efficacement aux questions auxquelles le traducteur ne trouve pas de réponses ou encore trouve des réponses divergentes dans les dictionnaires habituels. Il fournira ainsi au traducteur un outil sûr, de qualité, que celuici n'a ni le temps ni la capacité de forger lui-même et sans lequel il risque d'utiliser une langue échevelée et imprécise.

Alors, la terminologie pour quoi faire? Pour aider le traducteur, mais aussi pour protéger les langues de spécialité contre les fantaisies idiosyncrasiques de traducteurs pressés.

AMAL JAMMAL 